\title{
Stereoselective Synthesis of Vinylcyclopropanes from 2-Phenylsulfonyl-1,3-cyclohexadienes and Ketone Enolates or Nitrile Anion
}

\author{
Anna M. Ericsson, Niklas A. Plobeck and Jan-E. Bäckvall* \\ Department of Organic Chemistry, University of Uppsala, Box 531, 75121 Uppsala, Sweden
}

\begin{abstract}
Ericsson, A. M., Plobeck, N. A. and Bäckvall, J.-E., 1994. Stereoselective Synthesis of Vinylcyclopropanes from 2-Phenylsulfonyl-1,3-cyclohexadienes and Ketone Enolates or Nitrile Anion. - Acta Chem. Scand. 48: 252-257 () Acta Chemica Scandinavica 1994.

Michael addition of ketone enolates to 2-(phenylsulfonyl)-1,3-cyclohexadienes and subsequent cyclization affords vinylcyclopropanes in a highly stereoselective manner. In this process the enolate anion of the adduct displaces the allylic phenylsulfonyl group. The analogous reaction between the anion of benzyl cyanide and 2-(phenylsulfonyl)-1.3-cyclohexadiene afforded the corresponding vinylcyclopropane. An attempted synthesis of sesquicarene led to the sesquicarene derivative 3,endo-7-dimethyl-exo-7-(4-hydroxy-4-methylpentanoyl)-2-norcarene.
\end{abstract}

The organic chemistry of sulfones has undergone remarkable expansion over the last two decades and a number of synthetic transformations involving the sulfone functional group have been developed. ${ }^{1}$ A variety of versatile sulfone-containing synthons are known today and among those, 2-arylsulfonyl 1,3-dienes have recently attracted attention. ${ }^{2-7}$ Such 2-arylsulfonyl 1,3-dienes are useful Diels-Alder dienes, ${ }^{\text {6a.d,e,7 }}$ and, furthermore, they can be transformed into versatile synthetic intermediates via regioselective functionalization at either double bond. ${ }^{2 a, b, e, 6 b, c}$ Michael addition to these dienes is of particular interest since it produces an allylic sulfone, which can undergo further interesting functionalizations.

It is known that an allylic phenylsulfonyl group can act as a leaving group. ${ }^{1 \mathrm{~b}, \mathrm{c}, 8,9}$ A Michael addition of a carbon nucleophile to a 2-phenylsulfonyl 1,3-diene followed by intramolecular displacement of the allylic sulfonyl group would therefore be a possible route to vinylcyclopropanes [eqn. (1)]. In fact there is precedence for cyclization of an intermediate of type $\mathbf{B}$ to a vinylcyclopropane where $\mathbf{B}$ was generated via a different route. ${ }^{8}$

Vinylcyclopropanes occur as the structural unit in a number of natural products ${ }^{10}$ and they are also important synthetic intermediates. ${ }^{11}$ In connection with our previous studies on vinylcyclopropanes ${ }^{2 f, 12}$ and our general in- terest in 2-phenylsulfonyl 1,3-dienes ${ }^{2,6}$ we decided to study the possible synthetic route outlined in eqn. (1).

\section{Results and discussion}

Reaction of the enolates of ketones $\mathbf{2}, \mathbf{3}$, and $\mathbf{4}$ or the anion of nitrile 5 with 2-phenylsulfonyl-1,3-cyclohexadiene (1) at low temperature $\left(-78\right.$ to $\left.-25^{\circ} \mathrm{C}\right)$ afforded the corresponding Michael adducts. These adducts were isolated and characterized in several cases. When the initially formed Michael adduct was stirred at an elevated temperature $\left(20\right.$ to $\left.50^{\circ} \mathrm{C}\right)$ without prior quenching, cyclization to a vinylcyclopropane took place (Table 1). The cyclization, in which the sulfone acts as a leaving group, was highly stereoselective and gave only one diastereoisomer of the corresponding cyclopropane. It was established by NOE measurements and other NMR data (see the Experimental) that the isomer formed has the large group exo in products $6,7,8$, and 9 (Table 1). It is conceivable that the Michael adduct initially produced undergoes an intramolecular anion exchange $(\mathbf{A} \rightleftarrows \mathbf{B})$ followed by a cyclization with displacement of the sulfone. The high stereoselectivity in the formation of the cyclopropane to give the product with the large group exo can

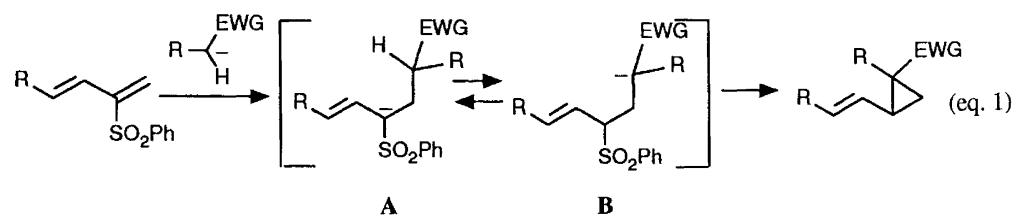


be explained by steric interaction in the transition state. Two approaches of the anion are possible one with the small group and one with the large group pointing towards the ring (Scheme 1). It is evident that steric interactions will be more severe in the latter approach.

Sesquicarene (10), is a hydrocarbon isolated from the essential oil of fruits of Schisandra chinensis Baill. ${ }^{13}$ This naturally occurring hydrocarbon contains a vinylic cyclopropane which we attempted to synthesize with our new procedure.

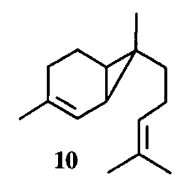

The strategy (Scheme 2) was to add the dianion of 6-hydroxy-6-methyl-3-heptanone ${ }^{14}(12)$ to the sulfonyl diene 11 and subsequently raise the temperature to promote cyclization. Reduction of the carbonyl group followed by elimination of water to give the most highly substituted double bond ${ }^{15}$ would lead to $\mathbf{1 0}$.

Addition of the dianion of $\mathbf{1 2}$ to $\mathbf{1 1}$ gave the corresponding Michael adduct 13. Attempts to cyclize the initially formed dianionic adduct simply by heating the reaction mixture prior to quenching failed. This is probably due to formation of a dianion in which the hydroxy group is always deprotonated. Apparently the alkoxide ion raises the $\mathrm{p} K_{\mathrm{a}}$ of the proton $\alpha$ to the carbonyl group more than that of the proton $\alpha$ to the sulfonyl group, ${ }^{16}$ and for this reason the anion required for cyclization was not formed. To avoid this effect the Michael adduct 13 was isolated and subsequently treated with one equivalent of base in THF. Under these conditions cyclization to vinylcyclopropane 14 occurred. The cyclization was again highly stereoselective. The anion responsible for the cyclization, which is in equilibrium with the two other possible anions, does have the $\mathrm{OH}$ group deprotonated in this case (Scheme 2). The yield obtained in THF was not satisfactory and therefore several other solvents or solvent systems were tried. Increasing the polarity by using DME decreased the yield of cyclized product and only a few percent of the product could be isolated. Reaction in

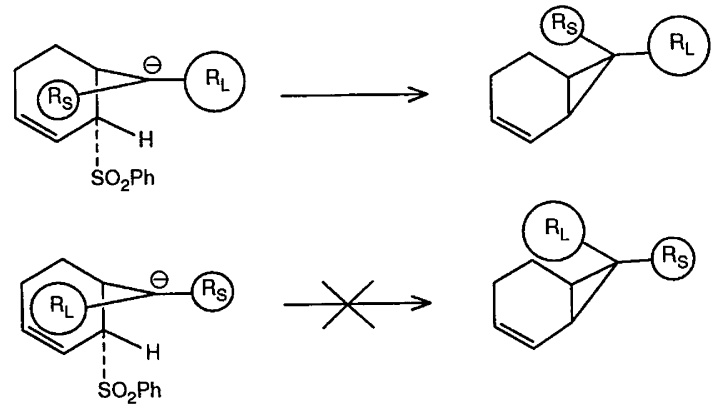

Scheme 1 . a less polar solvent increased the yield, and reaction in benzene resulted in an acceptable yield of the desired product (Table 2). This unexpected result is probably due to a different degree of cyclic hemiacetal formation in the Michael adduct. In the less polar solvent, benzene, the hemiacetal formation of the anion is suppressed compared with the situation in the more polar solvent THF. The analogous transformation of unsubstituted sulfonyl diene 1 into vinylcyclopropane 16 via 15 was also carried out under these reaction conditions (Table 2).

The next step was either to reduce the carbonyl group to a methylene group or to eliminate water. To avoid migration of the double bond to give an $\alpha, \beta$-unsaturated ketone we decided to reduce the carbonyl group first. Several procedures for reduction of carbonyl groups are known. ${ }^{17}$ Some of the methods tried include: (i) $\mathrm{N}_{2} \mathrm{H}_{4} \cdot \mathrm{H}_{2} \mathrm{O}, \mathrm{K}_{2} \mathrm{CO}_{3}-\left(\mathrm{HOCH}_{2} \mathrm{CH}_{2}\right)_{2} \mathrm{O} ;{ }^{18}$ (ii) thioacetal formation ${ }^{19 a, b}$ followed by Raney-Ni reduction; ${ }^{19 c}$ (iii) formation of a tosylhydrazone $\mathrm{e}^{20 \mathrm{a}}$ followed by $\mathrm{NaBH}_{3} \mathrm{CN}$ reduction. ${ }^{20 \mathrm{~b}}$ In all these cases clcavage of the cyclopropane occurred. To date we have not been able to reduce the carbonyl group to the required $\mathrm{CH}_{2}$ group. Apparently the carbonyl group is too activated ${ }^{11}$ and also too sterically hindered for successful reduction.

The present paper describes a procedure for the stereoselective transformation of 2-phenylsulfonyl-1,3-cyclohexadienes into vinylcyclopropanes. An attempted synthesis of sesquicarene (10) led to the sesquicarene derivative 14.

Table 1. One-step procedure. Formation of vinylcyclopropanes from conjugate addition of enolate and nitrile anions to 2-phenylsulfonyl 1,3-cyclohexadienes.

Entry $\begin{aligned} & \text { Sulfonyl } \\ & \text { diene }\end{aligned}$
Nucleophile
Cyclopropane

${ }^{a}$ Isolated yield after flash chromatography. 
Table 2. Two-step procedure. Formation of vinylcyclopropanes from conjugate addition of 6-hydroxy-6-methyl-3-heptanone to 2-phenylsulfonyl-1,3-cyclohexadienes.

Entry Sulfonyl diene Yield (\%) $)^{a}$

${ }^{a}$ Isolated yield after flash chromatography.

\section{Experimental}

General methods. NMR spectra were recorded either on a Varian XL-300 MHz or a Varian Unity $400 \mathrm{MHz}$ spectrometer: ${ }^{1} \mathrm{H}$ at $300 \mathrm{MHz}$ or $400 \mathrm{MHz}$ and ${ }^{13} \mathrm{C}$ at 74.5 $\mathrm{MHz}$ or $100.6 \mathrm{MHz}$, using $\mathrm{CDCl}_{3}$ as the solvent and tetramethylsilane $\left(0.0 \mathrm{ppm},{ }^{1} \mathrm{H}\right)$ and $\mathrm{CDCl}_{3}$ $\left(77.0 \mathrm{ppm},{ }^{13} \mathrm{C}\right)$ as internal standards. IR spectra were recorded on a Perkin-Elmer 1600 FT-IR spectrophotometer using a $0.1 \mathrm{~mm} \mathrm{KBr}$ cell with $\mathrm{CDCl}_{3}$ or $\mathrm{CCl}_{4}$ as the solvent. Only the strongest and structurally most important peaks $\left(v_{\max } / \mathrm{cm}^{-1}\right)$ are listed. Mass spectra were recorded on a Finnigan MAT INCOS 50 mass spectrometer. Elemental analyses were performed by Analytische Laboratorien, Engelskirchen, Germany. Reaction solvents were dried and distilled under nitrogen using standard procedures. Merck silica gel 60 (230-400 mesh) was employed for flash chromatography. 2-Phenylsulfonyl dienes
1 and $\mathbf{1 1}$ were prepared according to literature methods. ${ }^{\text {al.c.d }}$ Compounds 2-5 were purchased from $\mathrm{Al}-$ drich. 6-Hydroxy-6-methyl-3-heptanone (12) was prepared according to Ref. 14.

General procedure for the preparation of cyclopropanes (entries 1, 2 and 3, Table 1). A solution of lithium diisopropylamide (LDA) was prepared by addition of diisopropylamine $(0.25 \mathrm{ml}, 1.82 \mathrm{mmol})$ to an ice-cooled mixture of $n$-BuLi (1.14 ml, 1.6 M in hexane, $1.82 \mathrm{mmol})$ in dry THF $(4.13 \mathrm{ml})$. The solution was stirred for $30 \mathrm{~min}$. The ketone $(1.82 \mathrm{mmol})$ was then added dropwise at $-25^{\circ} \mathrm{C}$. The solution was stirred for $15 \mathrm{~min}$, then the temperature was raised to $50^{\circ} \mathrm{C}$ and a solution of the sulfonyl diene $1(200 \mathrm{mg}, 0.91 \mathrm{mmol})$ in THF $(1.00 \mathrm{ml})$ was added slowly $(12 \mathrm{~h})$ by use of a syringe pump. The reaction mixture was stirred at $50^{\circ} \mathrm{C}$ for the appropriate reaction time as indicated below. The reaction was quenched with

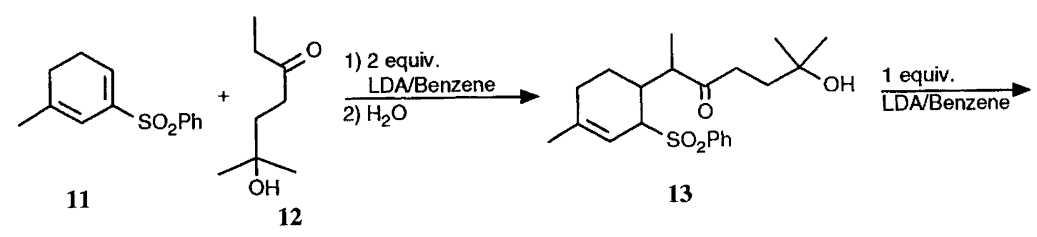<smiles>CC1=CC(=O)C(C(C)C(=O)CCC(C)(C)O)C(C(C)C(=O)CCC(C)(C)O)CC1C(=O)Oc1ccccc1</smiles><smiles></smiles><smiles>CC1=CC2CC(C)(C(=O)CCC(C)(C)O)C2CC1</smiles>

Scheme 2. 
$\mathrm{NH}_{4} \mathrm{Cl}(\mathrm{aq})$ and extracted with EtOAc $(\times 3)$. The combined organic extracts were washed with $\mathrm{H}_{2} \mathrm{O}(\times 2)$ and brine and dried $\left(\mathrm{MgSO}_{4}\right)$. Evaporation of the solvent and flash chromatography (EtOAc-hexane) gave the pure product.

endo-7-Methyl-exo-7-propionyl-2-norcarene (6). The reaction was stirred for 3 days after completed addition of the sulfonyl diene. The crude product was purified by flash chromatography (EtOAc-hexane $=10: 90)$ to give $92.5 \mathrm{mg}(62 \%)$ of cyclopropane 6. IR $\left(\mathrm{CDCl}_{3}\right): 3155$, 3032, 2979, 2937, 2880, 1678, 1459, 1379, 1350, 1218, 1191, 1124, $1032 \mathrm{~cm}^{-1}$; ${ }^{1} \mathrm{H}$ NMR: $\delta 5.83(\mathrm{dt}, J=10$, $\left.4.0 \mathrm{~Hz}, 1 \mathrm{H}, \mathrm{CH}_{2} \mathrm{CH}=\mathrm{CH}\right), 5.75(\mathrm{dm}, J=10 \mathrm{~Hz}, 1 \mathrm{H}$, $\left.\mathrm{CH}_{2} \mathrm{CH}=\mathrm{CH}\right), 2.55\left(\mathrm{q}, J=7 \mathrm{~Hz}, 2 \mathrm{H}, \mathrm{CH}_{3} \mathrm{CH}_{2}\right), 2.08$ $\left(\mathrm{m}, \quad 1 \mathrm{H}, \quad \mathrm{CH}_{2} \mathrm{CH}=\mathrm{CH}\right), \quad 1.97-1.86(\mathrm{~m}, 2 \mathrm{H}$, $\left.\mathrm{CH}=\mathrm{CHCH}, \quad \mathrm{CH}_{2} \mathrm{CH}_{2} \mathrm{CH}\right), \quad 1.86-1.72 \quad$ (m, $2 \mathrm{H}$, $\left.\mathrm{CH}_{2} \mathrm{CH}_{2} \mathrm{CH}, \mathrm{CH}_{2} \mathrm{CH}_{2} \mathrm{CH}\right), 1.65\left(\mathrm{~m}, 1 \mathrm{H}, \mathrm{CH}_{2} \mathrm{CH}_{2} \mathrm{CH}\right)$, $1.24\left(\mathrm{~s}, 3 \mathrm{H}, \mathrm{CH}_{3} \mathrm{C}\right), 1.03\left(\mathrm{t}, J=7 \mathrm{~Hz}, 3 \mathrm{H}, \mathrm{CH}_{3} \mathrm{CH}_{2}\right) ;{ }^{13} \mathrm{C}$ NMR: $\delta$ 211.2, 129.0, 123.0, 38.5, 32.5, 25.8, 25.2, 21.5, 15.6, 9.6, 8.1.

Irradiation of the protons of the 7-methyl group gave an NOE for the olefin protons, which is consistent only with the 7-methyl group being endo.

endo-7-Methyl-exo-7-benzoyl-2-norcarene (7). The reaction was stirred for $20 \mathrm{~h}$ after completed addition of the sulfonyl diene. The crude product was purified by flash chromatography $($ EtOAc-hexane $=10: 90)$ to give $127 \mathrm{mg}$ $(66 \%)$ of cyclopropane 7 . IR $\left(\mathrm{CDCl}_{3}\right): 3066,3031,2929$, $1665,1598,1580,1448,1265,1002 \mathrm{~cm}^{-1} ;{ }^{1} \mathrm{H}$ NMR: $\delta$ 7.77-7.71 (m, $2 \mathrm{H}), 7.54-7.39(\mathrm{~m}, 3 \mathrm{H}), 5.99-5.85(\mathrm{~m}$, $2 \mathrm{H}), 2.13(\mathrm{~m}, 1 \mathrm{H}), 2.03(\mathrm{~m}, 1 \mathrm{H}), 1.99-1.75(\mathrm{~m}, 4 \mathrm{H})$, $1.25(\mathrm{~s}, 3 \mathrm{H}) ;{ }^{13} \mathrm{C}$ NMR: $\delta$ 203.4, 137.3, 131.8, 128.7, 128.5, 128.2, 122.7, 37.8, 21.9, 21.8, 21.8, 15.4, 12.0 . Anal. calc. for $\mathrm{C}_{15} \mathrm{H}_{16} \mathrm{O}$ : C, 84.87; H, 7.60. Found: C, 84.67; H, 7.75.

exo-7-Benzoyl-2-norcarene (8). The reaction was stirred for $20 \mathrm{~h}$ after completed addition of the sulfonyl diene. The crude product was purified by flash chromatography (EtOAc-hexane $=5: 95)$ to give $115 \mathrm{mg}(64 \%)$ of cyclopropane 8. IR $\left(\mathrm{CDCl}_{3}\right)$ : 3038, 2932, 2852, 1660, 1598, $1580,1449,1415,1345,1292,1221,1039,1024 \mathrm{~cm}^{-1} ;{ }^{1} \mathrm{H}$ NMR: $\delta 7.96(\mathrm{~m}, 2 \mathrm{H}, \mathrm{ArH}), 7.58-7.42(\mathrm{~m}, 3 \mathrm{H}, \mathrm{ArH})$, 6.06 (ddd, $J=10,5,3 \mathrm{~Hz}, 1 \mathrm{H}, \mathrm{CH}_{2} \mathrm{CH}=\mathrm{CH}$ ), 5.64 (ddd, $\left.J=10,7,2 \mathrm{~Hz}, 1 \mathrm{H}, \mathrm{CH}_{2} \mathrm{CH}=\mathrm{CH}\right), 2.95(\mathrm{t}, J=4 \mathrm{~Hz}$, $1 \mathrm{H}, \mathrm{COCH}), 2.24(\mathrm{~m}, 1 \mathrm{H}, \mathrm{CH}=\mathrm{CHCH}), 2.19-1.70(\mathrm{~m}$, $\left.5 \mathrm{H}, \mathrm{CH}_{2} \mathrm{CH}_{2} \mathrm{CH}\right) ;{ }^{13} \mathrm{C}$ NMR: $\delta 198.6,137.9,132.6$, $128.4,127.9,126.0,125.4,30.3,27.4,25.3,20.7,17.8$; MS (70 eV): $m / z 198\left(M^{+}\right), 120,105,91,77$.

endo-7-Cyano-exo-7-phenyl-2-norcarene (9). To a solution of LDA (2.00 mmol) prepared as described above, was added benzyl cyanide $(230 \mu \mathrm{l}, 2.00 \mathrm{mmol})$ at $-78^{\circ} \mathrm{C}$ and the resulting mixture was stirred for $3 \mathrm{~h} .{ }^{9}$ 2-Phenylsulfonyl-1,3-cyclohexadiene $(440 \mathrm{mg}, 2.00 \mathrm{mmol})$ in THF $(1.00 \mathrm{ml})$ was added at $-78^{\circ} \mathrm{C}$ over $5 \mathrm{~min}$. The tem- perature was maintained at $-78^{\circ} \mathrm{C}$ for $3 \mathrm{~h}$ and was then allowed to reach $20^{\circ} \mathrm{C}$. After about $2 \mathrm{~h}$ a precipitate had formed and the reaction was quenched with $\mathrm{NH}_{4} \mathrm{Cl}$ (aq). Work-up as above, followed by flash chromatography $($ EtOAc-hexane $=10: 90)$ gave $250 \mathrm{mg}(64 \%)$ of 9 as a colorless oil, which crystallized on standing. M.p. 89$91^{\circ} \mathrm{C}$; IR $\left(\mathrm{CCl}_{4}\right): 2235 \mathrm{~cm}^{-1} ;{ }^{1} \mathrm{H}$ NMR: $\delta 7.40-7.20(\mathrm{~m}$, $5 \mathrm{H}, \mathrm{ArH}), 6.02(\mathrm{~m}, 2 \mathrm{H}, \mathrm{CH}=\mathrm{CH}), 2.40-2.07(\mathrm{~m}, 6 \mathrm{H}$, aliphatic-H); ${ }^{13} \mathrm{C}$ NMR: $\delta$ 136.7, 130.0, 128.8, 127.3, $125.1,120.6,119.3,30.0,29.1,28.6,20.5,17.0$.

The measured coupling constants ${ }^{3} J_{\mathrm{CH}}$ between the cyano group and the bridgehead protons were found to be $0 \mathrm{~Hz}$. This is consistent with the cyano group being endo. ${ }^{9,21}$ Anal. calc. for $\mathrm{C}_{14} \mathrm{H}_{13} \mathrm{~N}$ : C, 86.12; H, 6.71. Found: C, 85.95; H, 6.69.

4-(1,5-Dimethyl-5-hydroxy-2-oxohexyl)-1-methyl-3-(phenylsulfonyl)cyclohexene (13). A solution of LDA (115.1 mmol) was prepared as described above replacing THF with benzene. 6-Hydroxy-6-methyl-3-heptanonc (12) $(8.30 \mathrm{~g}, 57.56 \mathrm{mmol})$ was added to this solution at $0^{\circ} \mathrm{C}$ and the resulting mixture was stirred for $1 \mathrm{~h} .1-\mathrm{Me}-$ thyl-3-phenylsulfonyl-1,3-cyclohexadiene (11) (11.24 g, $47.97 \mathrm{mmol})$ in benzene $(90.5 \mathrm{ml})$ was added over $3 \mathrm{~h}$ by the use of a syringe pump and the reaction mixture was stirred for another $45 \mathrm{~min}$. The reaction was quenched with $\mathrm{NH}_{4} \mathrm{Cl}$ (aq). Purification of the crude product by flash chromatography $($ EtOAc-hexane $=30: 70)$ afforded $10.71 \mathrm{~g}(59 \%)$ of the pure adduct $(\mathbf{1 3})$ as two stereoisomers. IR $\left(\mathrm{CDCl}_{3}\right): 3600,3519,3069,2973,2935,1707$, $1668,1447,1374,1303,1249,1145,1085 \mathrm{~cm}^{-1}$. Major diastereoisomer: ${ }^{1} \mathrm{H}$ NMR: $\delta$ 7.88-7.82 (m, $\left.2 \mathrm{H}\right), 7.66$ $(\mathrm{m}, 1 \mathrm{H}), 7.60-7.52(\mathrm{~m}, 2 \mathrm{H}), 5.32(\mathrm{~m}, 1 \mathrm{H}), 3.43(\mathrm{~m}$, $1 \mathrm{H}), 2.82(\mathrm{~m}, 1 \mathrm{H}), 2.70-2.43(\mathrm{~m}, 3 \mathrm{H}), 1.96-1.48(\mathrm{~m}$, $6 \mathrm{H}), 1.73(\mathrm{~d}, J=1.2 \mathrm{~Hz}, 3 \mathrm{H}), 1.22(\mathrm{~s}, 3 \mathrm{H}), 1.22(\mathrm{~s}$, $3 \mathrm{H}), 1.08(\mathrm{~d}, J=7 \mathrm{~Hz}, 3 \mathrm{H}) ;{ }^{13} \mathrm{C}$ NMR: $\delta 214.2,144.4$, $137.5,133.6,129.2,128.9,111.5,70.1,64.6,47.5,36.5$, 35.4, 32.6, 29.5, 29.4, 26.6, 24.1, 20.1, 13.1. Minor diastereoisomer: ${ }^{1} \mathrm{H}$ NMR: $\delta 7.91-7.85(\mathrm{~m}, 2 \mathrm{H}), 7.65(\mathrm{~m}, 1 \mathrm{H})$, $7.60-7.52(\mathrm{~m}, 2 \mathrm{H}), 5.36(\mathrm{~m}, 1 \mathrm{H}), 3.66(\mathrm{~m}, 1 \mathrm{H}), 2.74-$ $2.38(\mathrm{~m}, 4 \mathrm{H}), 1.92-1.50(\mathrm{~m}, 6 \mathrm{H}), 1.74(\mathrm{~s}, 3 \mathrm{H}), 1.21(\mathrm{~s}$, $6 \mathrm{H}), 0.97(\mathrm{~d}, J=7 \mathrm{~Hz}, 3 \mathrm{H}) ;{ }^{13} \mathrm{C}$ NMR: $\delta 213.9,144.2$, $137.8,133.6,129.0,129.0,111.0,69.9,63.0,46.9,36.5$, $36.3,33.6,29.5,29.4,26.4,24.1,21.6,15.4$.

3,endo-7-Dimethyl-exo-7-(4-hydroxy-4-methylpentanoyl)-2norcarene (14). A solution of LDA $(27.5 \mathrm{mmol})$ was prepared as described above replacing THF with benzene and the adduct $13(10.00 \mathrm{~g}, 27.5 \mathrm{mmol})$ in benzene $(40.00 \mathrm{ml})$ was added at $0^{\circ} \mathrm{C}$. The temperature was raised to $80^{\circ} \mathrm{C}$ and the reaction mixture was stirred at this temperature for $16 \mathrm{~h}$. The reaction was quenched with $\mathrm{NH}_{4} \mathrm{Cl}$ (aq). Work-up as above followed by flash chromatography (EtOAc-hexane $=30: 70)$ afforded $4.55 \mathrm{~g}(70 \%)$ of the cyclopropane 14. IR $\left(\mathrm{CDCl}_{3}\right): 3604,3425,2972,2931$, 1668, 1448, 1386, 1372, $1131 \mathrm{~cm}^{-1}$; ${ }^{1} \mathrm{H}$ NMR: $\delta 5.44(\mathrm{~m}$, $1 \mathrm{H}, \mathrm{C}=\mathrm{CH}), 2.67\left(\mathrm{t}, \mathrm{J}=7 \mathrm{~Hz}, 2 \mathrm{H}, \mathrm{COCH}_{2} \mathrm{CH}_{2}\right), 2.02$ $\left(\mathrm{m}, 1 \mathrm{H}, \mathrm{CH}_{2} \mathrm{C}=\mathrm{CH}\right)$ 1.99-1.91 (m, $2 \mathrm{H}, \mathrm{C}=\mathrm{CHCH}$, 
$\left.\mathrm{CH}_{2} \mathrm{CH}_{2} \mathrm{CH}\right), \quad 1.83-1.56\left(\mathrm{~m}, 3 \mathrm{H}, \quad \mathrm{CH}_{2} \mathrm{CH}_{2} \mathrm{CH}\right.$, $\left.\mathrm{CH}_{2} \mathrm{CH}_{2} \mathrm{CH}, \mathrm{CH}_{2} \mathrm{CH}_{2} \mathrm{CH}\right), 1.75$ (t, J=7 Hz, $2 \mathrm{H}$, $\left.\mathrm{COCH}_{2} \mathrm{CH}_{2}\right), 1.70\left(\mathrm{~d}, J=0.8 \mathrm{~Hz}, 3 \mathrm{H}, \mathrm{CH}_{3} \mathrm{C}=\mathrm{CH}\right), 1.22$ [s, $\left.6 \mathrm{H}, \mathrm{C}\left(\mathrm{CH}_{3}\right)_{2} \mathrm{OH}\right], 1.20\left(\mathrm{~s}, 3 \mathrm{H}, \mathrm{C} \mathrm{H}_{3} \mathrm{CCO}\right) ;{ }^{13} \mathrm{C}$ NMR: $\delta 211.8,137.3,116.6,70.0,38.8,36.8,34.4,29.4$, $29.4,28.1,26.8,24.9,23.7,16.6,9.8$.

4-(1,5-Dimethyl-5-hydroxy-2-oxohexyl)-3-(phenylsulfonyl)cyclohexene (15). Prepared according to the same method as described above for 13. 3-Phenylsulfonyl-1,3-cyclohexadiene (1) (10.57 g, $47.97 \mathrm{mmol}$ ) was used instead of the sulfonyl diene 11. Work-up as above followed by flash chromatography (EtOAc-hexane $=40: 60)$ afforded $10.50 \mathrm{~g}(60 \%)$ of the pure adduct 15 as two diastereoisomers. IR $\left(\mathrm{CDCl}_{3}\right)$ : 3690, 3605, 3550, 3040, 2973, 2932, $1705,1602,1448,1373,1305,1146,1086 \mathrm{~cm}^{-1}$. Major diastereoisomer: ${ }^{1} \mathrm{H}$ NMR: $\delta$ 7.92-7.84 (m, $\left.2 \mathrm{H}\right), 7.66$ $(\mathrm{m}, 1 \mathrm{H}), 7.60-7.53(\mathrm{~m}, 2 \mathrm{H}), 6.15(\mathrm{dm}, J=10 \mathrm{~Hz}, 1 \mathrm{H})$, $5.59(\mathrm{dm}, J=10 \mathrm{~Hz}, 1 \mathrm{H}), 3.47(\mathrm{~m}, 1 \mathrm{H}), 2.88$ (quintet, $J=8 \mathrm{~Hz}, 1 \mathrm{H}), 2.71-2.45(\mathrm{~m}, 2 \mathrm{H}), 2.57(\mathrm{~m}, 1 \mathrm{H}), 1.96-$ $1.82(\mathrm{~m}, 3 \mathrm{H}), 1.80-1.65\left(\mathrm{~m}, 2 \mathrm{H}, \mathrm{CH}_{2}, \mathrm{AB}\right.$ part of ABXY-system), 1.65-1.48 (m, $2 \mathrm{H}$, include $\mathrm{OH}), 1.23(\mathrm{~s}$, $3 \mathrm{H}), 1.22(\mathrm{~s}, 3 \mathrm{H}), 1.10(\mathrm{~d}, J=7 \mathrm{~Hz}, 3 \mathrm{H}) ;{ }^{13} \mathrm{C}$ NMR: o $214.1,137.4,136.0,133.7,129.2,129.0,117.6,70.0$, $63.8,47.5,36.5,35.5,33.0,29.4,29.4,21.7,19.7,13.1$. Minor diastereoisomer: ${ }^{1} \mathrm{H}$ NMR: $\delta 7.92-7.86(\mathrm{~m}, 2 \mathrm{H})$, $7.66(\mathrm{~m}, 1 \mathrm{H}), 7.61-7.53(\mathrm{~m}, 2 \mathrm{H}), 6.16(\mathrm{~m}, 1 \mathrm{H}), 5.62$ $(\mathrm{m}, 1 \mathrm{H}), 3.67(\mathrm{~m}, 1 \mathrm{H}), 2.75(\mathrm{~m}, 1 \mathrm{H}), 2.66-2.45(\mathrm{~m}$, $3 \mathrm{H}), 1.96(\mathrm{~m}, 2 \mathrm{H}), 1.80-1.68(\mathrm{~m}, 3 \mathrm{H}), 1.55(\mathrm{~s}, 1 \mathrm{H}$, $\mathrm{OH}), 1.40(\mathrm{~m}, 1 \mathrm{H}), 1.21(\mathrm{~s}, 6 \mathrm{H}), 1.05(\mathrm{~d}, J=7 \mathrm{~Hz}, 3 \mathrm{H})$; ${ }^{13}$ C NMR: $\delta 213.9,137.7,135.9,133.7,129.1,129.1$, 117.2, 70.0, 62.3, 46.9, 36.5, 36.3, 34.1, 29.5, 29.4, 21.5, $21.1,15.4$.

endo-7-Methyl-exo-7-(4-hydroxy-4-methylpentanoyl)-2-norcarene (16). Prepared according to the same method as described above for 14, using adduct 15 in place of $\mathbf{1 3}$. The reaction mixture was stirred at $80^{\circ} \mathrm{C}$ for 2 days. $\mathrm{Pu}$ rification of the crude product by flash chromatography $($ EtOAc-hexane $=30: 70)$ afforded $3.48 \mathrm{~g}(57 \%)$ of the cyclopropane 16. IR $\left(\mathrm{CDCl}_{3}\right)$ : 3400, 3033, 2973, 1671, 1448, 1387, $1372 \mathrm{~cm}^{-1}$; ${ }^{1} \mathrm{H}$ NMR: $\delta 5.83(\mathrm{dt}, J=10,4 \mathrm{~Hz}, 1 \mathrm{H}$, $\mathrm{CH}=\mathrm{CHCH}), 5.74(\mathrm{dm}, J=10 \mathrm{~Hz}, 1 \mathrm{H}, \mathrm{CH}=\mathrm{CHCH})$, $2.67\left(\mathrm{t}, J=7 \mathrm{~Hz}, 2 \mathrm{H}, \mathrm{COCH}_{2} \mathrm{CH}_{2}\right), 2.07(\mathrm{~m}, 1 \mathrm{H}$, $\left.\mathrm{CH}_{2} \mathrm{CH}=\mathrm{CH}\right), \quad 1.97-1.86(\mathrm{~m}, 2 \mathrm{H}, \mathrm{CH}=\mathrm{CHCH}$, $\mathrm{CH}_{2} \mathrm{CH}_{2} \mathrm{CH}$ ), 1.84-1.70 (m, $\left.2 \mathrm{H}, \mathrm{CH}_{2} \mathrm{CH}_{2} \mathrm{CH}\right), 1.74$ (t, $\left.J=7 \mathrm{~Hz}, 2 \mathrm{H}, \mathrm{COCH}_{2} \mathrm{CH}_{2}\right), 1.68-1.55(\mathrm{~m}, 1 \mathrm{H}$, $\left.\mathrm{CH}_{2} \mathrm{CH}_{2} \mathrm{CH}\right), 1.25\left(\mathrm{~s}, 3 \mathrm{H}, \mathrm{CH}_{3} \mathrm{CCO}\right), 1.21[\mathrm{~s}, 6 \mathrm{H}$, $\left.\mathrm{C}\left(\mathrm{CH}_{3}\right)_{2} \mathrm{OH}\right] ;{ }^{13} \mathrm{C}$ NMR: $\delta 211.5,129.2,122.9,70.1$, $38.9,36.9,34.4,29.5,29.4,26.2$, 25.6, 21.5, 15.7, 9.7.

Irradiation of the protons of the 7-methyl group gave an NOE for the olefin protons, which is consistent only with the 7-methyl being endo. Anal. calc. for $\mathrm{C}_{14} \mathrm{H}_{22} \mathrm{O}_{2}$ : C, 75.63; H, 9.97. Found: C, 75.34; H, 9.82.

Acknowledgments. Financial support from the Swedish Natural Science Research Council is gratefully acknowledged.

\section{References}

1. (a) Schank, K. In: Klamann, D., Ed., Methoden Org. Chem. (Houben-Weyl), Stuttgart 1985, Band E 11, 1132; (b) Tanaka, K. and Kaji, A. In: Rappoport, Z. and Patai, S., Eds., The Chemistry of Sulphones and Sulphoxides, Wiley, New York 1988, p. 759; (c) Oae, S. and Uchida, Y. Ibid., p. 583; (d) Simpkins, N. S. Sulphones in Organic Synthesis, Pergamon, 1993.

2. (a) Bäckvall, J.-E. and Juntunen, S. K. J. Am. Chem. Soc. 109 (1987) 6396; (b) Bäckvall, J.-E. and Juntunen, S. K. J. Org. Chem. 53 (1988) 2398; (c) Andell, O. S. and Bäckvall, J.-E. Tetrahedron Lett. 26 (1985) 4555; (d) Bäckvall, J.-E., Juntunen, S. K. and Andell, O. S. Organic Synthesis 68 (1989) 148; (e) Bäckvall, J.-E., Nájera, C. and Yus, M. Tetrahedron Lett. 29 (1988) 1445; (f) Bäckvall, J.-E., Löfström, C., Juntunen, S. K. and Mattson, M. Tetrahedron Lett. 34 (1993) 2007.

3. (a) Back, T. G., Lai, E. K. Y. and Muralidharan, K. R. J. Org. Chem. 55 (1990) 4595; (b) Back, T. G., Lai, E. K. Y. and Muralidharan, K. R. Tetrahedron Lett. 30 (1989) 6481.

4. (a) Chou, S. S. P., Hsu, C. H. and Yeh, M. C. P. Tetrahedron Lett. 33 (1992) 643; (b) Chou, S. S. P. and Wey, S. J. J. Org. Chem. 55 (1990) 1270; (c) Chou, T. S. and Hung, S. C. Ibid. 53 (1988) 3020; (d) Padwa, A., Gareau, Y., Harrison, B. and Norman, B. H. J. Org. Chem. 56 (1991) 2713; (e) Padwa. A., Harrison, B. and Norman. B. H. Tetrahedron Lett. 30 (1989) 3259.

5. Posner, G. H. and Kinter, C. M. J. Org. Chem. 55 (1990) 3967.

6. (a) Bäckvall, J.-E., Löfström, C., Maffei, M. and Langer, V. Tetrahedron Lett. 33 (1992) 2417; (b) Bäckvall, J.-E., Ericsson, A. M., Plobeck, N. A. and Juntunen, S. K. Tetrahedron Lett. 33 (1992) 131; (c) Plobeck, N. A. and Bäckvall, J.-E. J. Org. Chem. 56 (1991) 4508; (d) Bäckvall, J.-E. and Plobeck, N. A. J. Org. Chem. 55 (1990) 4528; (e) Bäckvall, J.-E. and Rise, F. Tetrahedron Lett. 30 (1989) 5347.

7. (a) Inomata, K., Kinoshita, M., Takemoto, H., Murata, Y. and Kotake, H. Bull. Chem. Soc. Jpn. 51 (1978) 3341; (b) Cuvigny, T., Herve du Penhoat, C. and Julia, M. Tetrahedron 42 (1986) 5329; (c) Hoffmann, H. M. R., Weichert, A., Slawin, A. M. Z. and Williams, D. J. Tetrahedron 46 (1990) 5591; (d) Chou, T. and Hung, S.-C. J. Org. Chem. 53 (1988) 3020 .

8. (a) Julia, M. and Guy-Rouault, A. Bull. Soc. Chim. Fr. (1967) 1410; (b) Campbell, R. V. M., Crombie, L., Findley, D. A. R., King, R. W., Pattenden, G. and Whiting, D. A J. Chem. Soc., Perkin Trans. 1 (1975) 897; (c) Martel, J. and Huynh, C. Bull. Soc. Chim. Fr. (1967) 985.

9. Agawa, T., Yoshida, Y., Komatsu, M. and Ohshiro, Y. J. Chem. Soc., Perkin Trans. I (1981) 751.

10. (a) Handbook of Terpenoids, Dev, S., Ed., CRC, Boca Raton, FL 1982, Vol. 2; (b) Moore, R. E. Acc. Chem. Res. 10 (1977) 40; (c) Elliot, M. and Janes, N. F. Pyrethrum: the Natural Insecticides, Casida, J. E., Ed., Academic, New York 1973, p. 56; (d) Elliot, M. and Janes, N. F. Chem. Soc. Rev. 7 (1978) 473; (e) Arlt, D., Jautelat, M. and Lantzsch, R. Angew. Chem., Int. Ed. Engl. 20 (1981) 703.

11. Danishefsky, S. Acc. Chem. Res. 12 (1979) 66.

12. Bäckvall, J.-E., Vågberg, J. O., Zercher, C., Genêt, J. P. and Dennis, A. J. Org. Chem. 52 (1987) 5430.

13. (a) Nutting, W. H., Rapoport, H. and Machlis, L. J. Am. Chem. Soc. 90 (1968) 6434; (b) Otha, Y. and Hirose, Y. Tetrahedron Lett. (1968) 1251; (c) Corey, E. J. and Achiwa, K. Tetrahedron Lett. (1969) 3257; (d) Corey, E. J. and Achiwa, K. Tetrahedron Lett. (1969) 1837; (e) Corey, E. J. and Achiwa, K. Tetrahedron Lett. (1970) 2245; (f) Coates, R. M. and Freidinger, R. M. J. Chem. Soc., Chem. Commun. (1969) 871.

14. (a) Corey, E. J. and Enders, D. Chem. Ber. 111 (1978) 1337; 
(b) Harvey, S., Weisler, D. and Novotny, M. J. Chromatogr. 491(1) (1989) 27-29.

15. Posner, G. H., Shulman-Roskes, E. M., Oh, C. H., Carry, J.-C., Green, J. V., Clark, A. B., Dai, H. and Anjeh, T. E. N. Tetrahedron Lett. 32 (1991) 6489.

16. This may be due to formation of a cyclic hemiacetal anion, or to the larger charge separation obtained when the second anion is $\alpha$ to the sulfonyl group.

17. Larock, R. C. Comprehensive Organic Transformations, VCH New York 1989.

18. Paquette, L. A. and Annis, G. D. J. Am. Chem. Soc. 105 (1983) 7358.
19. (a) Paquette, L. A. and Ham, W. H. J. Am. Chem. Soc. 109 (1987) 3025; (b) Basu, N. K., Ghatak, U. R., Sengupta, G. and Dutta, P. C. Tetrahedron 21 (1965) 2641; (c) Ruff, A. and Reichstein, T. Helv. Chim. Acta 34 (1951) 70.

20. (a) Chamberlin, A. R., Liotta, E. L. and Bond, F. T. Organic Syntheses, Wiley, New York 1990, Collect. Vol. VII, p. 77; (b) Hutchins, R. O., Milewski, C. A. and Maryanoff, B. E. J. Am. Chem. Soc. 95 (1973) 3662.

21. Kingsbury, C. A., Durham, D. L. and Hutton, R. J. Org. Chem. 43 (1978) 4696.

Received August 12, 1993. 